\title{
The Need For and How To Evaluate Continuous Wave Immunity of Wireless Systems used in V2X Applications
}

\author{
Tim Claeys \\ M-Group \\ KU Leuven Bruges Campus \\ 8200 Bruges, Belgium \\ tim.claeys@kuleuven.be
}

\author{
Aleksandr Ovechkin \\ M-Group \\ KU Leuven Bruges Campus \\ 8200 Bruges, Belgium \\ aleksandr.ovechkin@kuleuven.be
}

\author{
Dries Vanoost \\ $M$-Group \\ KU Leuven Bruges Campus \\ 8200 Bruges, Belgium \\ dries.vanoost@kuleuven.be
}

\author{
Guy A. E. Vandenbosch \\ ESAT-Telemic \\ KU Leuven \\ 3001 Leuven, Belgium \\ guy.vandenbosch@kuleuven.be
}

\author{
Davy Pissoort \\ $M$-Group \\ KU Leuven Bruges Campus \\ 8200 Bruges, Belgium \\ davy.pissoort@kuleuven.be
}

\begin{abstract}
This paper describes an intial study of the susceptibility to EMI of the wireless technologies which are or will be used in Intelligent Transportation Systems (ITS). In recent literature reliability comparisons between the two newest Radio Access Technologies (RATs) IEEE802.11bd and NR V2X are starting to appear. Yet none of them consider single/multiple frequency EMI as a possible interference despite the fact that these can and will appear in reality. In this paper two simulation methods and a real-life test method, in a reverberation chamber, are proposed to evaluate the reliability of the RATs under these interferences. Single-frequency $\mathrm{CW}$ interference evaluation is possible for a limited number of combinations using simulations, but multifrequency CW simulations are nearly impossible to evaluate due to the large amount of possible combinations. Solutions are proposed to use a smaller test set of combinations. Evaluation of the RATs using simulations can be very useful, but will never reveal the full story how the RAT will react to interference. Therefore, a real-life test method in a reverberation room, or MIMO Antenna measurement system, is proposed trying to cover the full RAT protocol for a similar set of test combinations.

Index Terms-NR-V2X, IEEE802.11bd, Reverberation room, immunity characterization, simulations
\end{abstract}

\section{INTRODUCTION}

Fully autonomous vehicles of SAE level 5 are not yet commercially available. However, major technologies enabling autonomous driving are put into place. A major step in autonomous driving is the introduction of communication between Vehicles (V2V), Vehicle-to-Infrastructure (V2I),

The research leading to these results has received funding from the European Union's Horizon 2020 research and innovation programme under the Marie Skłodowska-Curie Grant Agreement No 812.788 (MSCA-ETN SAS) This publication reflects only the authors' view, exempting the European Union from any liability. Project website: http://etn-sas.eu/.
Vehicle-to-Person (V2P) and Vehicle-to-Network (V2N), or in the combined term Vehicle-to-Everything (V2X) (shown in Fig. 1) aiding cooperative automotive driving. The goal of $\mathrm{V} 2 \mathrm{X}$ is mainly aimed at making transport safer, more efficient and provide information to the vehicles. In general the term Intelligent Transport Systems (ITS) is used for the naming of transportation systems such as cars, agricultural vehicles, trucks, etc. In Europe a directive 2010/40/EU [1] has been written in order to create a framework to implement these kinds of transportation systems. Using delegate acts, the directive can be made more detailed. For example, a delegate act about the communication of these systems and their reliability, etc.

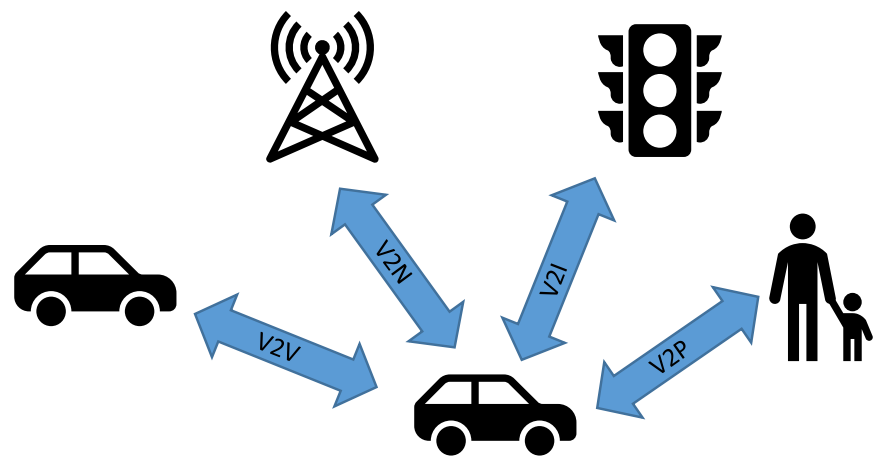

Fig. 1. Vehicle to Everything (V2X)

It is recommended, and expected, by the ECC that ITSs will use the frequency band of 5855-5925 MHz [2]. More specifically, the frequency band $5855-5875 \mathrm{MHz}$ will be used for non-safety applications, also part of the ISM band, while 
the safety applications are meant to use the frequency band 5875-5925 MHz.

To this date, two main Radio Access Technologies (RATs) are still competing to be chosen in Europe as the standard to be used for ITS communications. The first technology is based upon the well known and mature Wi-Fi technology IEEE802.11 and is known under different names like Dedicated Short Range Communications (DSRC), IEEE802.11p Wireless Access in Vehicular Environments (WAVE) [3], ETSI ITS-G5. Recently a new Task Group, Task Group IEEE802.11 (TGbd) has been started to improve the 802.11p V2X standard with newer technologies used in $802.11 \mathrm{~b}, \mathrm{~g}, \mathrm{n}$ and ac [4]. In Europe ITS-G5 [5], based on IEEE802.11p and standardized by ETSI EN 302663 [6], was proposed, by the European Commission, as the only technology in the delegate act of Cooperative-Intelligent Transport Systems C-ITS. Note that ITS-G5 is supported by companies as NXP, Toyota, etc. But it has been voted away by the European Union states [7] giving the opportunity to let the second main technology proof itself as a better or worse technology. This second type of technology is defined by the cellular communications group $3 \mathrm{GPP}$ and it is known as Cellular-V2X (C-V2X). Its first version is known as C-V2X Rel14 (4G) and is expected to have a new version in C-V2X Rel-16 (5G) or NR V2X. It is supported by the 5G Automotive Association (5GAA) and by big companies as BMW, Qualcomm and many others.

Naik et al. [4] presented and compared the newest RATs for V2X, IEEE802.11bd and NR-V2X, focussing and explaining the main items to to know about these two technologies' Media Acces Control (MAC) layer and physical (PHY) layer. In [8], the authors presented a reliability comparison between the same new RATs 802.11bd and NR-V2X, concluding that NR$\mathrm{V} 2 \mathrm{X}$ has a higher reliability for multiple scenarios and channel models, defined in [9], compared to IEEE802.11bd. Yet, they only tested the reliability of these RATs under Additive White Gaussian Noise. Which is, according to our honest opinion of this paper, not enough to conclude that either RAT is better than the other. What about the reliability under (un)-intentional EMI which certainly cannot always be considered as Additive White Gaussian Noise?

Obviously, the safety of autonomous vehicles greatly depends on these types of communications. Hence, dependability of those RATs is very important, not only against standard scenarios and noise but also against other signals. As EMI can be a major treat for the RATs, in-depth investigation of their robustness and resilience against EMI is definitely needed.

In the world of EMC, many types of unintentional interferences are known:

- Single-frequency continuous wave $(\mathrm{CW})$, expressed as a single sine wave

- Multi-frequency continuous waves (CWs), expressed as a sum of multiple sine waves

- Other wireless communication, either in or out of band.

- etc.

In a worst-case scenario, many of these interferences can even happen at the same time. Note that in this paper we will only focus on unintentional EMI and not on intentional EMI. This is left for future work. In this paper we describe how these RATs can be evaluated by either simulations or measurements for there basic immunity against single $\mathrm{CW}$, multiple $\mathrm{CWs}$ and other signals while keeping in mind the wireless channels and the possible movement of the vehicles.

In this paper we make suggestions on how to characterize the dependability of those RATs against different types of EMI. In Section II we specify which types of EMI are likely to occur in a certain scenario. Section III specifies the parameters for a single-frequency CW EMI wave in urban scenarios while Section IV specifies the parameters for multi-frequency $\mathrm{CW}$ EMI in urban scenarios. Section $\mathrm{V}$ describes a setup fitted in a reverberation chamber or MIMO antenna measurement system as the main proposed characterization. Section VI draws concluding remarks.

\section{Possible Unintentional Interference ScEnarios}

In this section, we expand the table given in [8] and also shown in Table I with likely EMI scenarios. In every subsection we try to define multiple parameters for each given EMI in all scenarios described in Table I.

\section{A. Rural and highway scenarios}

In a rural or highway scenario different types of simple and more complex channels are present. But in those environments we would assume that the number of possible unintentional interferers is relatively low and that interferers are not that close to the vehicles. Although interference is unlikely it could still happen or may happen more often in the future. The two most likely interferences are other RATs used by other vehicles or intra-EMI in the car itself. The latter of which should be tackled by the car manufacturers themselves. Interference between multiple RATs has already been discussed in [9] and is therefore not considered further in this paper.

\section{B. Urban scenarios}

In the urban scenarios, Line of Sight (LOS) and non-LOS are considered. Together with a higher number of electromagnetic scatterers, it leads to a more complex multipath scenario. Adding to this it can be assumed that a high density of other electronic devices is present leading to a higher chance of interference. Also we can consider either a standstill and a moving scenario. In this urban environment the following possible interferers are present:

- Out-Of-Band (OOB) emissions coming from the $5.8 \mathrm{GHz}$ ISM band from $5725 \mathrm{MHz}$ to $5875 \mathrm{MHz}$, which is close to the previsioned ITS frequency band. Although under control by the Radio Equipment Directive 2014/53/EU and more specifically specified in EN 330 440, OOB emissions are specified up to $\pm 250 \%$ of the occupied bandwidth from the centre frequency. This means that in a worst case example OOB emissions, for a $20 \mathrm{MHz}$ channel, can occur up to $5905 \mathrm{MHz}$. Beyond that frequency only spurious emissions remain which are limited to $30 \mathrm{dBm}$. There is no specification between $\pm 100 \%$ and 
TABLE I

CHANNEL MODEL PARAMETERS FROM [8]

\begin{tabular}{|l|l|l|l|}
\hline V2V Scenario & Power (dB) & Delay (ns) & Doppler (Hz) \\
\hline Rural LOS & {$\left[\begin{array}{llll}0 & -14 & -17\end{array}\right]$} & {$\left[\begin{array}{llll}0 & 83 & 183\end{array}\right]$} & {$\left[\begin{array}{llll}0 & 492 & -295\end{array}\right]$} \\
\hline Urban Approaching LOS & {$\left[\begin{array}{llll}0 & -8 & -10 & -15\end{array}\right]$} & {$\left[\begin{array}{llll}0 & 117 & 183 & 333\end{array}\right]$} & {$\left[\begin{array}{llll}0 & 236 & -157 & 492\end{array}\right]$} \\
\hline Urban Crossing NLOS & {$\left[\begin{array}{llll}0 & -3 & -5 & -10\end{array}\right]$} & {$\left[\begin{array}{llll}0 & 267 & 400 & 533\end{array}\right]$} & {$\left[\begin{array}{llll}0 & 295 & -98 & 591\end{array}\right]$} \\
\hline Highway LOS & {$\left[\begin{array}{llll}0 & -10 & -15 & -20\end{array}\right]$} & {$\left[\begin{array}{llll}0 & 100 & 167 & 500\end{array}\right]$} & {$\left[\begin{array}{llll}0 & 689 & -492 & 886\end{array}\right]$} \\
\hline Highway NLOS & {$\left[\begin{array}{llll}0 & -2 & -5 & -7\end{array}\right]$} & {$\left[\begin{array}{llll}0 & 200 & 433 & 700\end{array}\right]$} & {$\left[\begin{array}{llll}0 & 689 & -492 & 886\end{array}\right]$} \\
\hline
\end{tabular}

$\pm 250 \%$ of the occupied bandwidth. Since the Effective Isotropic Radiated Power (EIRP) can be $14 \mathrm{dBm}$ we would assume that in the worst case the EIRP between $\pm 100 \%$ and $\pm 250 \%$ of the occupied bandwidth can be somewhere in between $14 \mathrm{dBm}$ and $-30 \mathrm{dBm}$ EIRP. Note that for Short Range Devices below $1 \mathrm{GHz}$, OOB emissions should be below $0 \mathrm{dBm}$. This kind of interferer is further referred to as an OOB interferer and can actually be approximated as a band limited Additive White Gaussian Noise (AWGN) due to the randomness of the interfering communication channel. General AWGN immunity has already been tested by [8].

- According to CISPR 32 peak radiated emissions of multimedia equipment can be $80 \mathrm{~dB} \mu \mathrm{V} / \mathrm{m}$ peak at $3 \mathrm{~m}$ or $-16 \mathrm{dBm}$ EIRP and $60 \mathrm{~dB} \mu \mathrm{V} / \mathrm{m}$ on average at a distance of $3 \mathrm{~m}$ or $-35 \mathrm{dBm}$ EIRP for frequencies above $1 \mathrm{GHz}$. The same limits apply for the generic standard 610006-4 (industrial environments). This interferer is further referred to as a general interferer. The type of noise coming from a general interferer can show itself in different types. As for example: one or multiple CWs, AWGN and time intermittent varieties of those EMI.

- Beside the EMI coming from a device that has been measured in EMC testing we should also consider EMI stemming from the fact that devices are not always to spec or can break down and emit more noise than originally measured. For example: in time due to different environmental parameters, cables get loose and cause sparks which in its turn cause very high electromagnetic fields over a large frequency range. Those type of interferers can be referred to as excessive interferers.

More types of possible interferers can be thought of than these. Yet all of them come down to either wideband random noise, single/multiple $\mathrm{CW}$ or a combination of them. They can be time-intermittent and have different spectral characteristics. Characterizing the immunity RATs against wideband noise sources is always performed by using AWGN [8] and is not further analysed in this paper. On the other hand, single or multiple CW as an interferer for V2X RATs has never been considered so far, while it is most certainly of big importance.

\section{SinglE CW IMMUNITY SIMULATIONS IN AN URBAN ENVIRONMENT}

The transmitted power of any RAT for V2X will fall under a certain legislation which is not known yet. Though, current proof of concepts assume a transmitted power of $21 \mathrm{dBm}$ EIRP [10]. Hence, in this paper it is assumed to have a transmitting power of $21 \mathrm{dBm}$ EIRP. In what follows two cases are defined in which a single $\mathrm{CW}$ falls into either a standstill or a moving scenario.

\section{A. Standstill scenario}

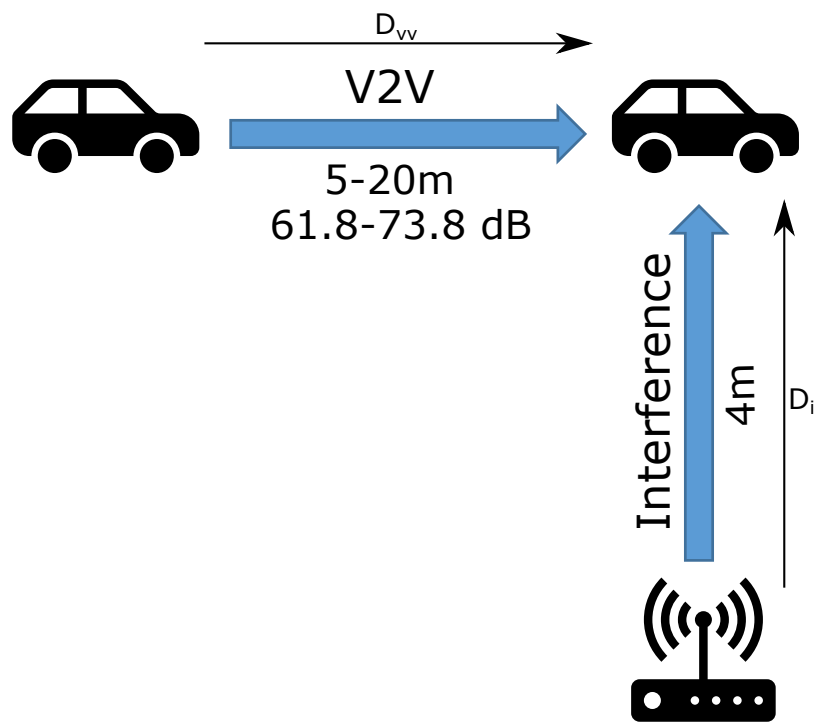

Fig. 2. Standstill scenario

The standstill scenario, shown in Fig 2, assumes that the distance $D_{i}$ between an interferer and a vehicle is $4 \mathrm{~m}$. The same vehicle receives a packet from another standstill vehicle at a distance $D_{v v}$ between 5 to $20 \mathrm{~m}$. Assuming no reflections (simple Friis equation) between the interferer and the receiving vehicle results in a $59.9 \mathrm{~dB}$ path loss. The path loss between the vehicles will be at least $61.8 \mathrm{~dB}$ at $5 \mathrm{~m}$ or maximum $73.8 \mathrm{~dB}$ at $20 \mathrm{~m}$, again assuming no reflections. The received interference power at the receiving vehicle results in $-75.9 \mathrm{dBm}$ (the maximum power of a general interferer, $-16 \mathrm{dBm}$, lowered with the path loss) while the received communication packet power is between $-40.8 \mathrm{dBm}$ and $52.8 \mathrm{dBm}$ (the maximum power of $\mathrm{V} 2 \mathrm{X}$ communications, $21 \mathrm{dBm}$, lowered with the path loss). Resulting in a Signal to Interferer Ratio (SIR,(1)) of 23.1 to $35.1 \mathrm{~dB}$. But, if we look at path loss measurements in urban environments as done by Nilsson et al. [11] a difference with the free space path loss of $\pm 20 \mathrm{~dB}$ can occur. Hence the possible SIR can be expanded to 3.1 to $55.1 \mathrm{~dB}$. It is also a good idea to expand it even further to a minimum of $0 \mathrm{~dB}$, just to build in sufficient margins. Hence, 
the power of an interference can possibly be as large as the $\mathrm{V} 2 \mathrm{~V}$ communication packet.

$$
\mathrm{SIR}=20 \log _{10}\left(\frac{P_{\text {signal }}}{P_{\text {interferer }}}\right)
$$

Since the $\mathrm{CW}$ interference, defined as $C W=$ $A \sin (2 \pi f t+\theta)$, can come from any direction at any frequency $f$, it is proposed to use the $\mathrm{CW}$ interference at many different phase angles $\Theta$ (for example 360 angles between 0 and $359^{\circ}$ ) and many frequencies $f$ in the RATs frequency band. Both RATs use Orthogonal Frequency Division Multiplexing (OFDM) to cope with multipath fading. This OFDM technique has shown to be susceptible to single and multiple CW [12]. The exact frequency difference between the $\mathrm{CW}$ and the subcarrier of the OFDM subchannel also has a large effect [12] due to spectral leakage [13]. Hence, we propose to test the RAT with at least 10 different frequencies per OFDM subchannel. In case the RAT has 64 OFDM subchannels, a total number of 9216000 simulations are needed, considering 360 different phases, $64 \cdot 10$ different frequencies and 40 different SIR levels. Through careful scripting and the use of matrices, simulations can be parallelized and reduced in time. The simulation time can be further reduced by using multiple processes on a CPU or GPUs. Yet, it is not possible to simulate every scenario. It should on the other hand be possible to expose many of the weak points of both RATs.

\section{B. Moving scenario}

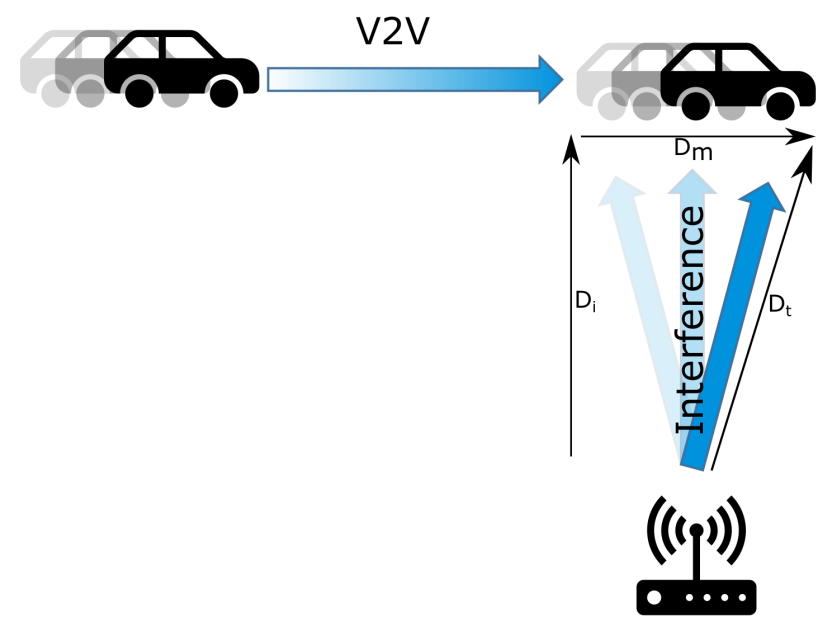

Fig. 3. Moving scenario

The previous considers an EMI interferer falling in to a vehicle which is not moving at all. Yet in most cases vehicles are also moving. Furthermore we take the case that both vehicles move at approximately $108 \mathrm{~km} / \mathrm{h}$ or $30 \mathrm{~m} / \mathrm{s}$ according to the doppler shift of $591 \mathrm{~Hz}$ at $5.9 \mathrm{GHz}$ shown in Table I. Also considering that the end-to-end latency (time between action of the first vehicle untill reaction of the second vehicle) of a wireless communication packet should be less than $100 \mathrm{~ms}$, we will assume a total packet length of $10 \mathrm{~ms}$ in the worst case (similar to the packet length of IEEE802.11p). In that $10 \mathrm{~ms}$ a vehicle would move over a distance of $D_{m}=0.3 \mathrm{~m}$. Hence, the distance between the interferer and the moving vehicle changes. From these parameters one could calculate the possible attenuation and phase shift over $10 \mathrm{~ms}$ of the interference to the vehicle. Again assuming a free space loss and a minimum distance of again $D_{i}=4 \mathrm{~m}$ between interferer and vehicle, a difference of only $0.006 \mathrm{~dB}$ occurs using equation (2). This difference is too small to take into account.

$$
\operatorname{Diff}=20 \log _{10}\left(\frac{\lambda}{4 \pi D_{i}}\right)-20 \log _{10}\left(\frac{\lambda}{4 \pi \sqrt{D_{i}^{2}+\left(\frac{D_{m}}{2}\right)^{2}}}\right)
$$

The phase on the other hand changes more significantly. The instantaneous distance between the interferer and the vehicle $D_{t}$ changes from $4 \mathrm{~m}$ to $4.0028 \mathrm{~m}$. Resulting in a wavelength difference of $5.51 \%$ or $20^{\circ}$ approximately. The phase change $\theta_{\text {change }}$ due to the moving vehicle can be described as:

$$
\theta_{\text {change }}=\frac{\sqrt{D_{i}^{2}+(v t)^{2}}}{\lambda} 2 \pi
$$

with $v$ the speed of the vehicle and $\lambda$ the wavelength of the $\mathrm{CW}$ interference. The phase difference is shown in Fig 4.

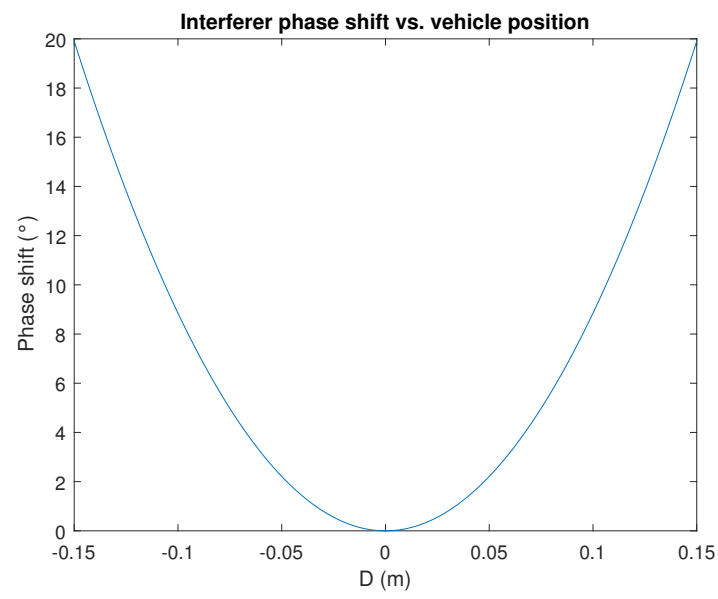

Fig. 4. EMI phase shift

A phase that changes in time during a single packet of $10 \mathrm{~ms}$ can also be looked at as a change in frequency of the $\mathrm{CW}$ during that single packet of $10 \mathrm{~ms}$. So, another way of looking at this is using the Doppler effect. The perceived velocity $v_{p}$ of the vehicle can be derived from the angle $\phi$ between the interferer and the vehicle itself by using $v_{p}=v \cos (\phi)$ with $\phi=a \tan \left(\frac{D_{i}}{D_{m}(t)}\right)$. The perceived frequency of the vehicle can then be defined as:

$$
f_{p}=f\left(\frac{c+v_{p}}{c}\right)
$$


In the case under consideration, this is limited to a frequency change of only $\approx 44 \mathrm{~Hz}$ in total. Yet it would still be enough for the $\mathrm{CW}$ interference to hop from the one OFDM subchannel to the other making $\mathrm{CW}$ removal techniques as shown in [12] possibly more difficult. Allthough probably not of big influence in comparison with a stable $\mathrm{CW}$ interference, this effect should definitely be considered and evaluated. So instead of adding $\sin (2 \pi f t+\theta)$ as an interference to the RAT, $\sin \left(2 \pi f t+\theta+\frac{\sqrt{D_{i}^{2}+(v t)^{2}}}{\lambda} 2 \pi\right)$ should be added, with all proposed parameters from the standstill scenario and now also including different vehicle speeds $v$.

\section{MUltiple CW IMMUNiTY SimUlations IN AN URBAN ENVIRONMENT}

Multiple CWs originating from multiple general interferers can interfere with the RATs. It is not unlogical to think that multiple interferers occur at the same time from a random direction on different frequencies, with each of them having a different SIR. If all of these interferers occur in the same OFDM sub channel and constructively interfere, the total maximum SIR increases to $-20 \mathrm{~dB}$ for ten interferers. Ideally one would test the RAT against as much as possible interferers, lets assume 10, with 360 possible incoming angles, and at least 10 frequencies per OFDM channel. Yet it would result in a total $360^{10}(\cdot 64 \cdot 10)^{10} \cdot 40 \approx 1.26 \cdot 10^{55}$ simulations for an RAT with 64 OFDM sub channels. Assuming that the simulation time per RAT packet would be $1 \mathrm{~ms}$ it would still take $8 \cdot 10^{44}$ years to simulate all possibilities.

Several possibilities exist to reduce the simulation time. A first solution is to exploit the weak points in the RAT with one single CW and use those to apply multiple CW to the RAT. For example, 20 frequencies are very susceptible at a single $\mathrm{CW}$ for 2 specific angles. Exploiting these weak points with 10 $\mathrm{CWs}$ at the same time would result in $\approx 2 \cdot 10^{13}$ simulations, which is still way too much. Reducing the number of interferers would certainly reduce the number of simulations. We therefore recommend by in this case applying two interferers on the 20 'weak frequencies' which in its turn would lead to lesser 'super weak frequencies', hopefully. In a next simulation one could continue with three interferers on those 'super weak frequencies', and so on. Eventually it would expose the major weak points of each RAT.

Another method would be to let a randomiser choose the different parameters of different interferers. It would be necessary though, to produce the correct probabilities of amplitude, phase and frequency. Similar simulations have been performed in [14]-[16].

This still just applies to a standstill urban scenario as shown in Fig 5 for only 4 interferers. Imagine the number of possible simulations that need to be performed when the vehicles are moving!

\section{REAL-WORLD TESTING OF RATS UNDER CW INTERFERENCE}

Allthough simulations are very useful to characterize, compare and improve the RATs, it is still not a real-life test and

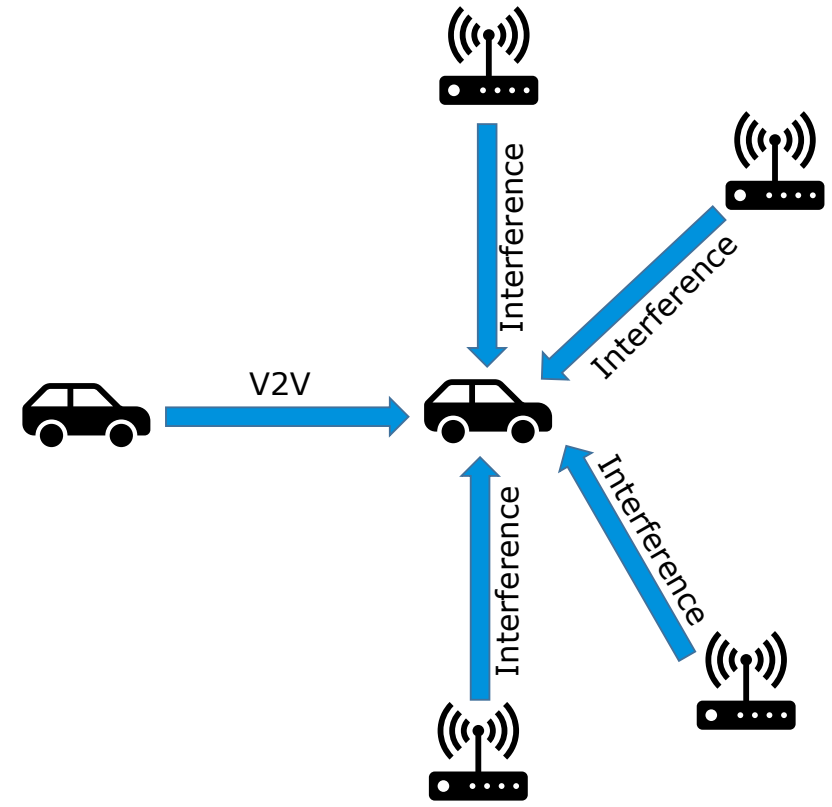

Fig. 5. Multiple interferers in a standstill scenario

there always remains the question if simulations are accurate enough to represent real-life conditions. Hence, the authors of this papers also suggest to characterize these RATs on a real implementation, either implemented on a real ASIC for current RATs or on software defined radios. Building a test site with moving vehicles in the middle of multiple interferers is not an easy task. Instead of the latter, the real implemented RATs could be placed in a reverberation chamber that simulates an urban environment similar to the work of Genender et al. [17].

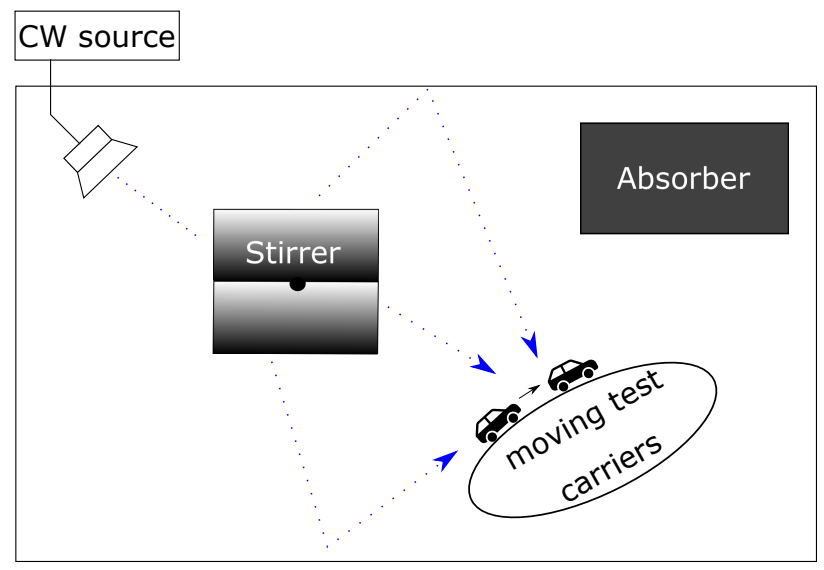

Fig. 6. Reverberation room test setup

In this reverberation room, two test carriers (the size of a small printed circuit board) can be placed which hold the implementation of the RAT. The test carriers, shown as two vehicles in Fig 6, could be placed at a standstill or be moving with some kind of mechanical system. Ideally the test carriers would be moving at a speed of $30 \mathrm{~m} / \mathrm{s}$, which is in theory not impossible, but could pose to be a real challenge in practice. 
Packet error rates could be monitored live by using a wireless protocol which uses another frequency band and is able to cope with the multipath and fast fading of the moving test carriers. Since most reverberation rooms are not big enough to provide a $5 \mathrm{~m}$ transmission path between two test carriers, an attenuator can be used to simulate the same path loss.

Since the reverberation room is too reverberant in its own and the power delay profile (PDP) is too large [18], absorbers need to be added to have a PDP similar to an urban environment. A single or multiple $\mathrm{CW}$ source can be amplified or attenuated to produce a SIR up to $0 \mathrm{~dB}$ at the test carriers. By slowly moving the stirrer while the test carriers are communicating, multiple paths are created simulating multiple interferers. Together with the generation of a single $\mathrm{CW}$, multiple $\mathrm{CWs}$ or even an $\mathrm{OOB}$ emission, a very harsh EM environment can be created, really characterizing the dependability of possible RATs. Note that this real-life test will not cover more combinations of parameters than simulations. However if properly done, it might be a better approximation of reality due to the use of real RAT implementations.

Another proposed real-life test setup is the use of a MIMO OTA chamber as proposed in [19]. In this test chamber, multiple antennas in a circle around the system under test can be used to generate any kind of pre defined EM environment. Changing the system under test by the moving test carriers can be used to mimic very specific and harsh EM environments. Allthough this EM environment is very controllable it will also not cover all possible combinations of parameters.

\section{CONCLUSION}

Research in the topic of comparing the two newest Radio Access Technologies (RAT) for the automotive industry is still in its infancy. Only a couple of papers deal with the comparison between IEEE802.11bd and NR V2X. The decision on what RAT eventually should be used is still not made in Europe and should be based on specific and concrete research results. Not only should the reliability of those RATs be evaluated against the usual Additive White Gaussian Noise but also to other forms of EMI, like single-frequency or multifrequency continuous waves. In this paper, two simulation strategies for single and multiple $\mathrm{CW}$ interference have been proposed. Allthough very useful, simulations never tell us the full story. Therefore a real-life test method in a reverberation chamber is proposed. Choosing which RAT to be used in the automotive industry should also partially depend on these test results. It should also be noted that testing $100 \%$ of all possible scenarios, parameters or combinations is impossible in simulations as well as real-life testing. But through a careful research and exploiting weak points of the RATs, a specific set of combinations can be made, which should be enough to choose one specific RAT for future autonomous driving. The authors want to emphasize the need for a thorough EMI risk management plan because there will always be a possibility that communication is lost and could lead to a safety risk.

\section{REFERENCES}

[1] European Commission, "Directive 2010/40/eu, on the framework for the deployment of intelligent transport systems in the field of road transport and for interfaces with other modes of transport," 2010. [Online]. Available: https://eur-lex.europa.eu/legalcontent/EN/TXT/PDF/?uri=CELEX:32010L0040\&from=EN

[2] ECC, "ECC recommendation (08)01 use of the band 5855-5875 mhz for intelligent transport systems (its)," 2015. [Online]. Available: https://www.ecodocdb.dk/download/798c1836-20c6/REC0801.pdf

[3] "IEEE standard for information technology- local and metropolitan area networks- specific requirements- part 11: Wireless LAN medium access control (MAC) and physical layer (PHY) specifications amendment 6: Wireless access in vehicular environments," IEEE Std 802.11p2010 (Amendment to IEEE Std 802.11-2007 as amended by IEEE Std 802.11k-2008, IEEE Std 802.11r-2008, IEEE Std 802.11y-2008, IEEE Std 802.11n-2009, and IEEE Std 802.11w-2009), pp. 1-51, July 2010.

[4] G. Naik, B. Choudhury, and J. Park, "IEEE 802.11bd 5g nr v2x: Evolution of radio access technologies for v2x communications," IEEE Access, vol. 7, pp. 70 169-70 184, 2019.

[5] A. K. N. S. TÜV, "Its-g5 technology - a fact sheet."

[6] ETSI, "Intelligent transport systems (its); its-g5 access layer specification for intelligent transport systems operating in the $5 \mathrm{ghz}$ frequency band," 52019.

[7] F. Y. Chee, "Eu opens road to $5 \mathrm{~g}$ connected cars in boost to bmw, qualcomm," July 2019. [Online]. Available: https://www.reuters.com/article/us-eu-autos-tech/eu-opens-road-to$5 \mathrm{~g}$-connected-cars-in-boost-to-bmw-qualcomm-idUSKCN1TZ11F

[8] W. Anwar, A. Traß1, N. Franchi, and G. Fettweis, "On the reliability of nr-v2x and IEEE 802.11bd," in 2019 IEEE 30th Annual International Symposium on Personal, Indoor and Mobile Radio Communications (PIMRC), Sep. 2019, pp. 1-7.

[9] M. Kahn, "IEEE802.11 regulatory sc dsrc coexistence tiger team v2v radio channel models," Feb 2014.

[10] Qualcom, "c-v2x-technology-overview." [Online]. Available: https://www.qualcomm.com/media/documents/files/c-v2x-technologyoverview.pdf

[11] M. Nilsson, C. Gustafson, T. Abbas, and F. Tufvesson, "Path loss and shadowing model for multilink vehicle-to-vehicle channels in urban intersections," Sensors (Basel), Dec 2018.

[12] K. Fors, E. Axell, S. Linder, and P. Stenumgaard, "On the impact of cw interference on 5g nr," in 2019 International Symposium on Electromagnetic Compatibility - EMC EUROPE, Sep. 2019, pp. 10491054.

[13] T. Claeys, D. Vanoost, J. Peuteman, G. A. E. Vandenbosch, and D. Pissoort, "An iterative interpolated dft to remove spectral leakage in timedomain near-field scanning," IEEE Transactions on Electromagnetic Compatibility, vol. 60, no. 1, pp. 202-210, Feb 2018.

[14] M. Magdowski, S. V. Tkachenko, and R. Vick, "Coupling of stochastic electromagnetic fields to a transmission line in a reverberation chamber," IEEE Transactions on Electromagnetic Compatibility, vol. 53, no. 2, pp. 308-317, May 2011.

[15] D. A. Hill, "Plane wave integral representation for fields in reverberation chambers," IEEE Transactions on Electromagnetic Compatibility, vol. 40, no. 3, pp. 209-217, Aug 1998.

[16] J. Lannoo, A. Degraeve, D. Vanoost, J. Boydens, and D. Pissoort, "Effectiveness of inversion diversity to cope with emi within a twochannel redundant system," in 2018 IEEE International Symposium on Electromagnetic Compatibility and 2018 IEEE Asia-Pacific Symposium on Electromagnetic Compatibility (EMC/APEMC), May 2018, pp. 216220.

[17] E. Genender, C. L. Holloway, K. A. Remley, J. M. Ladbury, G. Koepke, and $\mathrm{H}$. Garbe, "Simulating the multipath channel with a reverberation chamber: Application to bit error rate measurements," IEEE Transactions on Electromagnetic Compatibility, vol. 52, no. 4, pp. 766-777, Nov 2010.

[18] D. Senic and K. A. Remley, "Measurements of wireless devices in reverberation chambers," in 2018 IEEE Symposium on Electromagnetic Compatibility, Signal Integrity and Power Integrity (EMC, SI PI), July 2018, pp. 1-31.

[19] B. Sayler, "Understanding MIMO OTA Testing: Simple solution to a complex test," in International CTIA Wireless 2011, March 2011. 